\title{
The Design and Analysis of a Muffler Based on Virtual.lab
}

\author{
Dongwei Wang \\ Xiamen University Tan Kah Kee College, Fujian, China
}

\begin{abstract}
In this paper, we analyzed the muffler acoustic performance by the finite element method and Virtual.lab. We changed the simple expansion chamber muffler size and expansion ratio related, expansion cavity length, and the number of expansion chambers effectively to improve the muffler. Through muffler design complexity and numerical simulation we analyzed the impact of external factors such as temperature, flow rate, etc. for muffler performance.
\end{abstract}

Keywords-noise elimination; exhaust muffler; acoustic simulation

\section{INTRODUCTION}

Noise pollution has become an important issue that can not be ignored in today's society, and its harmful to human beings in the following three aspects.

(1) damage to human health.

(2) affect people's work, life, and sleep.

(3) reduce people's work and learning efficiency.

Engine exhaust noise is one of the main sources of noise car noise, the current installation of exhaust muffler controls exhaust noise is the most direct and effective way. So it has a very important practical significance to study the acoustic properties of the muffler. Study of Automotive Exhaust Muffler traditional design method is mainly rely on the experience, which is very time-consuming, laborious, expensive. Three-dimensional numerical method to accurately simulate the exhaust muffler performance while also taking into account a variety of external factors, and it is an effective way to predict performance exhaust muffler and improve design.

\section{EXPANSION MUFFLER ANALYSIS}

The muffler is usually composed of a simple muffler unit, and the main structure of the muffler is composed of the expansion, the inner tube, the perforated plate and so on.

As the engine exhaust noise spectrum is low frequency, and there is a certain medium and high frequency noise, so the design of automobile exhaust muffler should be in the middle and low frequency which has a good performance. Because of the effect of the muffler in the middle and low frequency, it is often used to control the exhaust noise.

\section{A. Muffler Structure}

The exhaust noise power matching the reference engine exhaust muffler noise frequency spectrum, mainly concentrated in less than $2500 \mathrm{~Hz}, 2500-5000 \mathrm{~Hz}$. It also has a certain strength, the percentage is small, and the sensitive hearing frequency is about $3000 \mathrm{~Hz}$. For the amount of calculation, the calculated frequency range is set to $20-3000 \mathrm{~Hz}$, and the step size is $10 \mathrm{~Hz}$. The entity grid unit length should be between 13mm, type of hexahedral mesh grid.

Based on the above experimental research and conditions, the design of a composite muffler, which is composed of a plurality of expansion chambers, insert pipes and perforated plates. The main parameters are as follows:

Expansion ratio: $\mathrm{m}=16$; insert tube diameter: 25mm; diameter of internal intubation: 20mm; expansion chamber diameter: 100mm; expansion cavity number: 4; expansion cavity length: 70, 80, 80, 70; perforation plate thickness: 4mm. As shown in Figure I.

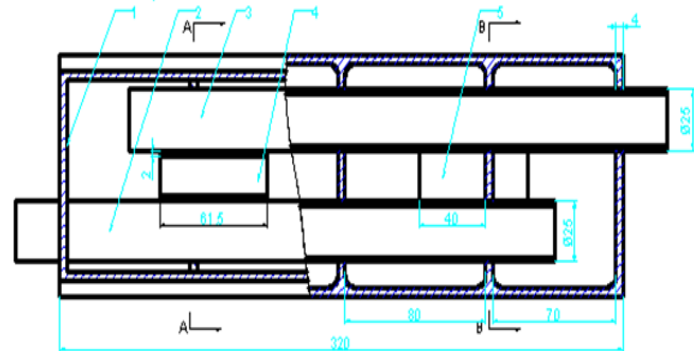

FIGURE I. MUFFLER THREE-DIMENSIONAL MODEL

\section{B. Determination of the Expansion Ratio}

Single stage expansion muffler is the simplest structure, and its transmission loss is:

$$
T L=10 \lg \left[1+0.25\left(\mathrm{~m}-\frac{1}{m}\right)^{2} \sin ^{2} k L\right]
$$

In the formula (1), $\mathrm{M}$ is the expansion ratio, $\mathrm{R}$ is the radius of the expansion chamber; $\mathrm{L}$ is the length of the expansion chamber; $\mathrm{R}$ is the radius of the inlet tube; $\mathrm{K}$ is the wave number, determined by the acoustic frequency.

Attention should be paid to the muffler design:

As the expansion ratio increases, the amount of noise attenuation increases. In order to increase the low-frequency 
noise, the expansion ratio is required. In practical problems, due to the constraints of objective conditions, the expansion ratio is not as big as possible, generally take $9<\mathrm{m}<16$, the maximum not more than 20 , the minimum is not less than 5 .

In order to reduce the influence of the frequency, in the practical application, it is usually to choose the appropriate insert tube, which can improve the effect of the noise reduction. Because of the influence of frequency, and the $\mathrm{M}$ is too large to consider the layout of the structural design, in fact, the use of two rooms or a number of expansion chamber portfolio to replace the single room.

In order to improve the effect of medium and high frequency noise, the muffler is divided into several parts. The expansion chamber section area is relatively small, can increase the upper limit frequency. Moreover, the inlet pipe and the outlet pipe are biased, so that the sound wave can not directly pass through the expansion chamber, and the utility model can play a certain role of noise elimination.

The cross-sectional area is too large, too large space when installed, when the expansion ratio to a certain extent, there will be a high frequency of failure. Therefore, we need to consider the size of the expansion chamber cross-sectional area [1].

The main research object of this paper is the design of the middle and low frequency muffler, combined with the conclusions of the above study, tentative muffler $m=16$.

According to the theoretical formula, the length of the expansion chamber should be:

$$
L=\frac{1}{4} \frac{\mathrm{c}}{f}
$$

By the formula (2) it can be seen that the lower the frequency of $\mathrm{F}$, the larger the expansion chamber length $\mathrm{L}$.

In order to study the relationship between the length of the expansion chamber and the transmission loss, the L was 100 , 150 and $200 \mathrm{~mm}$, respectively, and the expansion ratio of $\mathrm{m}=16$ (according to the expansion ratio formula, $\mathrm{R}=100 \mathrm{~mm}, \mathrm{r}=25 \mathrm{~mm}$ ) was determined. The transmission loss is calculated by Virtual.lab simulation, and the results are shown in the following Figure II:

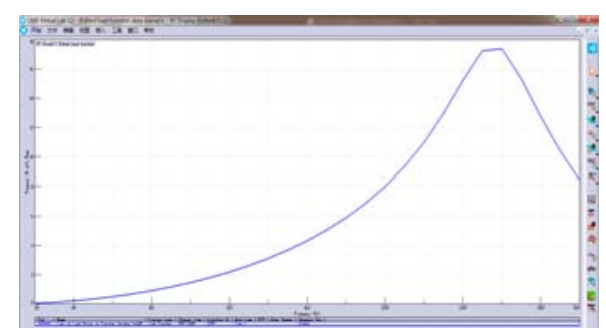

FIGURE II. THE TRANSMISSION LOSS WHEN THE EXPANSION CHAMBER IS 100MM

From Figure 1 it can be obtained that the peak transmission loss curve of different length is almost no change; and with the increase of the length, the frequency has not been eliminated, but moved to the low frequency direction. Changing the length of the expansion chamber can change the frequency, the center frequency and the bandwidth of the transmission loss.

The effect of expansion chamber number:

In order to study the relationship between the number of expansion chambers and the transmission loss, the length of the expansion chamber was $300 \mathrm{~mm}$, and the expansion ratio was $\mathrm{m}=16 \quad(\mathrm{R}=100 \mathrm{~mm}, \mathrm{r}=25 \mathrm{~mm})$. The number of expansion chambers was 1, 2 and 3, respectively. The transmission loss is calculated by Virtual.lab simulation, and the results are shown in the following Figure III:



From Figure 2, the following conclusions can be drawn:

With the increase of the number of expansion chamber, the numerical value of the transmission loss increases. The larger the number of expansion chamber, the lower the frequency, the higher the performance of high-frequency noise.

The transmission band of the transmission loss curve shown in the graph becomes wider and smaller by the number of frequencies.

The influence of the shape of the cross sectional area:

When the volume is constant, the shape of the cross section has no influence on the acoustic performance of the muffler. Therefore, in the design of the muffler, the cross-sectional shape can be installed in accordance with the chassis of the vehicle space to choose. So in this paper, we use circular section to design muffler.

The effect of intubation on the muffler performance

Changing the intubation depth of insertion in the cavity it can change the acoustic performance of the original muffler, theoretically, when intubation in the cavity is inserted into the expansion chamber is equal to the length of $1 / 2$, can be eliminated by frequency $\mathrm{n}$ is odd, when the inner cannel is inserted into a length equal to the length of the expansion chamber $1 / 4$, can be eliminated by frequency n even part, so the theory perspective, in the end of the muffler expansion type will be equal to the length of the cannel is inserted into the expansion chamber of the length of the $1 / 2$, the other end of the long tube insertion is the expansion cavity length of $1 / 4$, you can get there by frequency the muffler [2]. 


\section{Acoustic Finite ElEment AnAlysis}

As the design uses an expansion muffler, so the muffler theoretical calculation should be used to calculate the noise reduction, the calculation process is as follows:

Division of grid entities, select hexahedral mesh, dividing the cell size is $2 \mathrm{~mm}$, the partition result as shown in Figure IV.

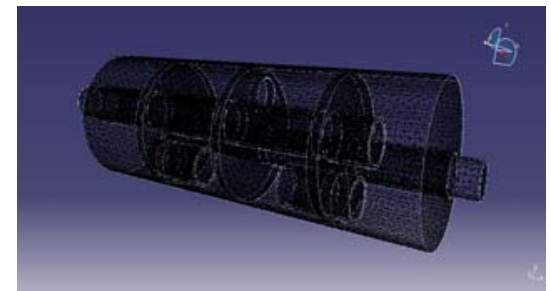

FIGURE IV. ACOUSTIC FINITE ELEMENT MESH

The propagation velocity of sound in a material and the density of the material is as shown in Figure V. Information can be obtained, the speed of sound in the air is $340 \mathrm{~m} / \mathrm{s}$, and the density of the fluid medium is $1.225 \mathrm{~kg} / \mathrm{m}^{3}$.

In acoustics meshes, we need to define the entrance and exit of sound to select the grid envelope opening here.

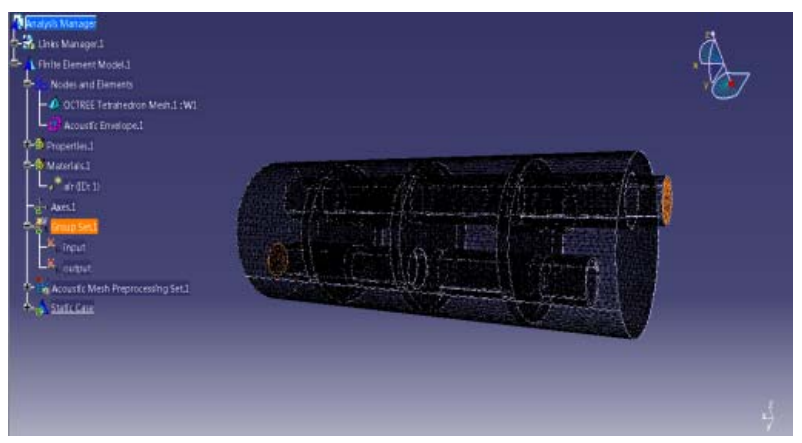

FIGURE V. DEFINES THE INLET AND OUTLET

In order to calculate the transmission loss of the muffler, it is necessary to define a boundary condition of the unit velocity at the inlet end.

The muffler is connected to the outlet of atmosphere; the sound through the export of travel directly to the atmosphere, because of the acoustic finite element mesh, the wall without any treatment, sound propagation to the exit of the wall will all bounce back. In order to simulate the effect of sound reflection at the exit, you can define a fully absorptive property at the exit, as shown in figure 3-12. The sound absorption properties, can be used in acoustic impedance or acoustic admittance defined for air outlet in the muffler acoustic impedance is the definition of absorption boundary, voice to export is not reflected.

Calculate the sound pressure distribution of the muffler, as shown in Figure V. The design can be drawn from the previous design, where the calculation of frequency $20 \mathrm{HZ} 3000 \mathrm{HZ}$, the calculation step is $10 \mathrm{HZ}$.

By updating the model data and calculating the distribution of sound pressure distribution, we can get the 20 3000HZ. Define the I/O input and output points, and see the inlet and outlet sound pressure response, the unit will be changed to $\mathrm{dB}$.
Frequency response function curve can be obtained as shown in Figure VI.

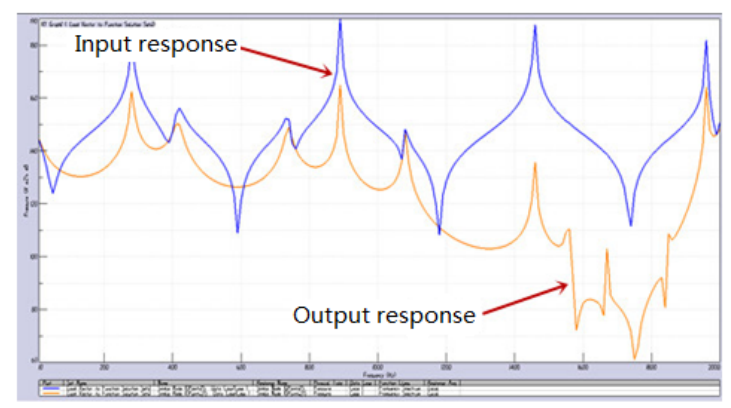

FIGURE VI. FREQUENCY RESPONSE CURVE

The transmission loss curve can be obtained according to the formula of transmission loss, and the transfer loss unit is $\mathrm{dB}$, and the transmission loss is shown in Figure VII.

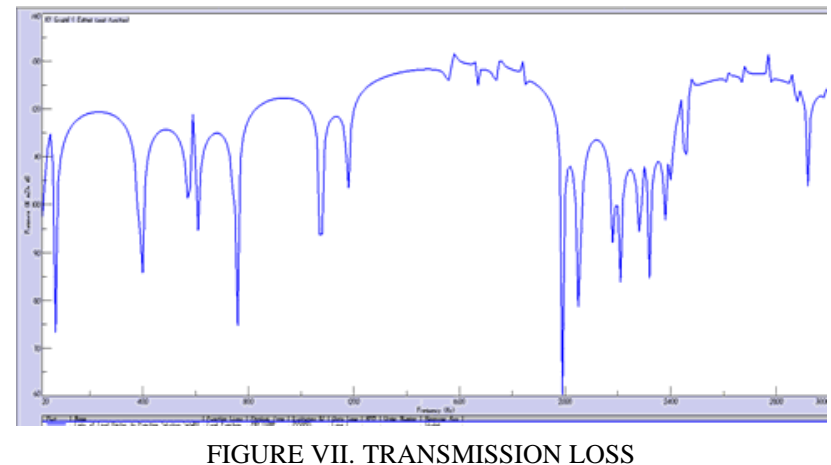

From the transmission loss curve 3-16 can be concluded that the muffler in the low frequency of the amount of noise can keep a good amount of noise in the high frequency has a good performance, but in the $2000 \mathrm{~Hz}$ when the amount of only $60 \mathrm{db}$.

Extracting the sound pressure reprogram is of $2000 \mathrm{~Hz}$. As shown in Figure VIII, it is obvious that the sound pressure at the inlet and outlet of the intake pipe is high.

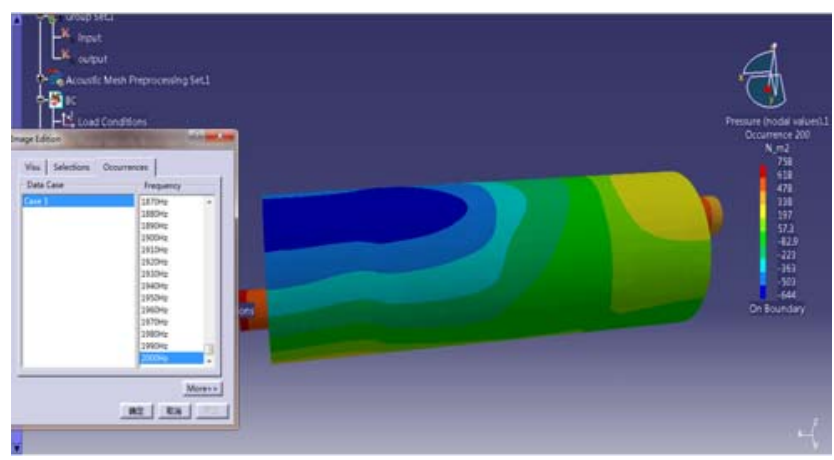

FIGURE VIII. SOUND PRESSURE DISTRIBUTION

\section{REFERENCES}

[1] Li Zenggang, Zhan Feng.Virtual.lab Acoustics acoustic simulation of advanced applications [M]. Beijing: National Defense University press, 2010 
[2] Yuan Qihui. Simulation study of automobile exhaust muffler based on [D]. Chongqing: Chongqing Jiaotong University, 2013 (in Chinese), Virtual_Lab

[3] An Ping. Material forming technology [M]. Publishing House: Science Press, 2008

[4] Liu Huiling. Environmental noise control [M]. Publishing House: Harbin Institute of Technology press, 2002-10s

[5] [Zhou Xinxiang. Noise control technology and its new progress [M]. Publishing House: Metallurgical Industry Press, 2007-7.

[6] Wei Zhiyong. Industrial noise and vibration control technology [M]. Publishing House: China labour and Social Security Publishing House, 2010-10.

[7] Wu Zongze, Luo Shengguo. Mechanical design curriculum design manual [M]. Publishing: Higher Education Press, 1999

[8] Yuan Changming. Cloud. Chinese dietary composition. Noise and vibration control technology of [M]. Publishing: Metallurgical Industry Press, 2007-7.

[9] Sheng Meiping Wang Minqing. Sun Jincai. Noise and vibration control technology base (Second Edition) [M]. published: Science Press, 200712.

[10] Ying Huaijiao. Vibration and noise of modern technology [M]. Publishing: Aviation Industry Press, 2005-10..

[11] Liu Weijun. Rapid prototyping technology and its application [M] Publishing House: Mechanical Industry Press, 2005-1.

[12] Fan Chunhua. Zhao Jianfeng.. rapid prototyping technology and its application $[\mathrm{M}]$. Publishing House: Publishing House of electronics industry, 2009-3. 